\title{
Article \\ Simultaneous Excitation of Helium by Means of an Electron and a Photon: A Joined Experimental and Theoretical Study
}

\author{
Imane Ajana ${ }^{1}\left(\right.$, Driss Nehari ${ }^{2}$, Driss Khali1 ${ }^{1}$, Abdelmalek Taoutioui ${ }^{3}\left(\mathbb{D}\right.$, Hicham Agueny ${ }^{4}(\mathbb{D}$ and \\ Abdelkader Makhoute $1,5, *$ (D) \\ 1 Physique du Rayonnement et des Interactions Laser-Matière, Faculté des Sciences, Moulay Ismail University, \\ Meknes 50070, Morocco; ajanaimane@gmail.com (I.A.); drisskhalil@hotmail.com (D.K.) \\ 2 Department of Physics, EMAFI Team, Faculté Polydisciplinaire de Khouribga, Sultan Moulay Slimane \\ University, Khouribga 25000, Morocco; driss.nehari@uhp.ac.ma \\ 3 Institute for Nuclear Research (ATOMKI), Bem tér 18/c, 4026 Debrecen, Hungary; \\ abdelmalek.taoutioui@atomki.hu \\ 4 Department of Physics and Technology, Allegt. 55, University of Bergen, N-5007 Bergen, Norway; \\ hicham.agueny@uib.no \\ 5 Faculté des Sciences, CP. 231, Campus Plaine, Université Libre de Bruxelles (U.L.B.), 1050 Brussels, Belgium \\ * Correspondence: makhoute@netcourrier.com
}

Citation: Ajana, I.; Nehari, D.; Khalil,

D.; Taoutioui, A.; Agueny, H.;

Makhoute, A. Simultaneous

Excitation of Helium by Means of an Electron and a Photon: A Joined

Experimental and Theoretical Study. Atoms 2021, 9, 67. https://doi.org/ $10.3390 /$ atoms 9030067

Academic Editor: David D. Reid

Received: 3 August 2021

Accepted: 14 September 2021

Published: 17 September 2021

Publisher's Note: MDPI stays neutral with regard to jurisdictional claims in published maps and institutional affiliations.

Copyright: (c) 2021 by the authors. Licensee MDPI, Basel, Switzerland. This article is an open access article distributed under the terms and conditions of the Creative Commons Attribution (CC BY) license (https:// creativecommons.org/licenses/by/ $4.0 /)$.

\begin{abstract}
We report on a joined experimental and theoretical study of differential cross-sections resulting from inelastic scattering of a monoenergetic electron by helium atoms in the presence of an intense carbon dioxide laser. In particular, we measured the signals of the scattered electrons during the simultaneous electron-photon excitation of $\mathrm{He}{ }^{1} \mathrm{P}$ state for the first three microseconds of the laser pulse. The signals were measured for an incident electron energy of $45 \mathrm{eV}$ and showed a structure that emerged at small scattering angles. The latter was found to be sensitive to the nature of the transferred photons, as well as the intensity of the laser field. The experimental findings were supported by quantum calculations based on the second-order Born approximation in which the correlated electron-electron interactions were taken into account.
\end{abstract}

Keywords: simultaneous electron-photon excitation; differential cross-sections; second-order Born approximation

\section{Introduction}

Electron scattering in the presence of laser fields plays a key role in understanding the basic mechanisms behind light-induced nonlinear processes in matter, such as high-order harmonic generation (HHG) and nonsequential double ionization (NDS). These processes constitute the basis of attosecond science [1,2].

The characteristic features that distinguish this class of elementary collision physics is the presence of three overlapping beams, which involve a photon, a monoenergetic electron, and a target. This combination has the advantage of enabling a high degree of control of atomic and molecular dynamics. In addition, the electron scattering process forms the basis of various processes in strong light-matter interaction [1,2]. A particular example is the HHG process, in which the electron after being ionized by a laser field is driven back and rescatters off the parent ion. Because of the presence of the oscillating laser field, the rescattered electron can emit photons. Here, the scenario repeats itself every half-cycle of the laser field, thus resulting in the emission of coherent high-frequency radiation $[3,4]$.

Another example is related to the NSD of helium atom by means of a low laser pulses (i.e., a few terawatts) in an experiment [5]. Here, the ionized electron is driven back by the laser field and rescatters by the helium ion, thus kicking out the second electron. Once again, the electron scattering process plays here a vital role in uncovering the origin of the mechanisms underlying the ionization of the second electron, which has yet to be completely understood. These examples illustrate the relevance of electron scattering 
processes in the presence of laser fields in providing new insights into the basic mechanisms in coherent light-matter interactions, and thus, further theoretical developments should be encouraged [6].

An interesting aspect of electron scattering in the presence of a laser field is the simultaneous electron-photon excitation (SEPE) process, in which the target is simultaneously excited by means of a monoenergetic electron and a photon. The complexity of this process in an experiment is related to the precise alignment of the three beams (i.e., electron and photon beams and the target), in addition to being focused and synchronized. This in turn has limited experimental investigations of the process. The first experiment of SEPE was performed by Mason and Newell [7,8] for a helium target using a $\mathrm{CW} \mathrm{CO}_{2}$ laser field with very low intensities in the range of $10^{4}-10^{5} \mathrm{~W} \mathrm{~cm}^{-2}$. Later on, additional experiments were improved using laser intensities up to $10^{8} \mathrm{~W} \mathrm{~cm}^{-2}[9,10]$, which were subsequently followed by the work of Luan et al. [11], in which higher intensities of the order $10^{10} \mathrm{~W} \mathrm{~cm}-2$ of a Nd-YAG-type laser were introduced. On the other hand, most of the experimental works of laser-assisted electron scattering using $\mathrm{CO}_{2}$ lasers applied to different noble gas targets were performed by Wallbank and coworkers [12-19].

On the theoretical side, most of the calculations were performed on the basis of the first-order Born approximation in which the atomic target involved either a single active electron or two active electrons [20-22]. Although this approximation is valid only at high electron energies, it has been shown to provide new insights into the inelastic scattering process assisted by laser fields, in particular in the regime where the perturbation theory for laser-atom interactions breaks down. For instance, it has been shown that the computed differential cross-sections encode information about the avoided crossing induced by a resonant laser field [23], which in turn can be controlled by varying the properties of the laser fields, as well as those of the monoenergetic electron beam [24,25]. At a low electron energy, the second-order Born approximation is required in order to take into account the additional effects such as the exchange effects [26,27], which are necessary for the precise comparison with an experiment. Additional methods are based on the R-matrix Floquet approach $[28,29]$ and the Born-Floquet method $[23,30]$.

In general, atoms had been excited from their ground states to higher states through collisions with electrons and/or photons. However, it has been demonstrated that excitation can also occur through the "simultaneous" impact of an electron and one or many photons. The laser introduces some parameters (laser photon energy, intensity, polarization ...) that influence the collisional process. Studies of this simultaneous electron-photon process for the prototype of a helium atom, in which the two correlated electrons were taken into account, were performed by Dunseath et al. [28,29]. However, here, the calculations were limited to bound-bound transitions, and only 11 states of helium were involved in the scattering process; thus, ionization was neglected. Furthermore, the collisional process has been studied for an incident electron beam of $22 \mathrm{eV}$. A similar study for the case where the internal structure of the atom does not change after the collision was presented in our former paper [31]; the process corresponds to elastic scattering in the free-free radiative transition.

This work was motivated by the strong interest in the simultaneous excitation process, which offers wider possibilities for controlling the outcome of the scattering process, as it involves both the properties of the photon and the incident electron beams. We thus present in this paper a joined experimental and theoretical study of the simultaneous excitation of helium by a monoenergetic electron and photon beams. We measured the signals of the scattered electrons for the first three microseconds of the laser pulse. We used an incident electron beam of $45 \mathrm{eV}$, which limited the scattering angle to $35^{\circ}$. We show that the recorded signals encoded a structure at a small scattering angle, which in turn was found to be sensitive to the nature of the absorbed/emitted photon process, as well as the intensity of the laser field. A direct comparison with the calculations based on the second-order Born approximation showed a good agreement with the experiment except at a very small scattering angle, in which the structure that emerged in the measured signal 
was absent. Additional calculations based on the Kroll-Watson approximation are also provided for further discussion.

The main body of the paper is organized as follows: In Section 2, we present the experimental setup, and in Section 3, we describe our theoretical method based on the second-order Born approximation. The theoretical results are compared with the experimental data in Section 4. Section 5 concludes the paper. Atomic units (a.u.) are used throughout this paper.

\section{Experimental Setup}

We give a brief description of the experimental setup; more details were reported by Wallbank and Holmes [12]. The apparatus consisted of three main parts, which were in the same differentially pumped vacuum chamber: the incident beam of electrons and the scattered beam of electrons constituted the spectrometer, and the beam of helium atoms was formed by a pulsed supersonic valve.

In this setup, an electron beam was produced by accelerating electrons considered to be emitted from a sharp tungsten filament and focusing them onto a $127^{\circ}$ cylindrical deflector. The electrons scattered by the atomic target were decelerated and refocused into a double hemispherical sector deflector for the energy selection and then became detected with an electron multiplier after reacceleration. In the present experiment, the spectrometer was operated such that the energy resolution was $55-70 \mathrm{meV}$. This resolution depended on the alignment of the incident electrons through the spectrometer. The calibration of the electron beam energy was achieved using the ${ }^{2} P_{\frac{3}{2}}$ argon resonance at $11.098 \mathrm{eV}$.

For the scattering angles $(6,9,12,15,20,25,30,35)^{\circ}$, the incident electron beam was fixed in the vector parallel to the laser polarization. We could not go further than $35^{\circ}$ as the count rate of the scattered electrons became too weak to be measured. The pressure in the vacuum chamber during the measurement was $10^{-6}$ mbar. The spectrometer design allowed reaching a high angular resolution of the detected electrons $\approx 2^{\circ}$; the divergence of the incident beam was neglected in the calculation of the errors.

The radiation was furnished by a pulsed $\mathrm{CO}_{2}$ TEA laser (at a wavelength of $10.6 \mu \mathrm{m}$ ) operating in multilongitudinal mode. The polarization of the laser beam was achieved by inserting a $\mathrm{NaCl}$ Brewster's angle polarizing element into the laser cavity. The polarizing element decreased the maximum output energy of the laser from $5 \mathrm{~J} /$ pulse to $3.4 \mathrm{~J} /$ pulse. The laser was focused in the scattering region to produce a field with an estimated maximum intensity of $4 \times 10^{8} \mathrm{~W} \mathrm{~cm}^{-2}$. The duration of the pulse was $3 \mu \mathrm{s}$.

The data were collected and analyzed separately during the first, second, and third microsecond. The analysis provided information about the dependency of the intensity of the cross-section. During the first microsecond, the average intensity was about $10^{8} \mathrm{~W} / \mathrm{cm}^{2}$ for the first $250 \mathrm{~ns}$ only and decreased quickly to $10^{7} \mathrm{~W} / \mathrm{cm}^{2}$. It remained roughly at the same magnitude for the rest of the pulse. The data were accumulated with a $200 \mathrm{~ns}$ time resolution for each pulse. To perform this analysis, the laser was triggered externally by a fiber optic and delay unit connection in the counter. We used a beam splitter in order to reflect a weak portion of the laser after the scattering region onto a photon drag detector to record the profile of the laser as a function of time (see page 2 of [15]). The laser was operated at a frequency of about $7 \mathrm{~Hz}$ and aligned by using a He-Ne visible laser.

\section{Theoretical Description}

We present here a short description of our theoretical approach to deal with the electron's impact excitation of the helium atom in the presence of a laser field. A detailed description can be found in our previous works [32-34]. The scenario we considered here can be simplified into the following form:

$$
H e\left(1^{1} S\right)+e^{-}\left(E_{k_{i}}, \mathbf{k}_{i}\right)+\ell \hbar \omega \rightarrow H e^{*}\left(2^{1} P\right)+e^{-}\left(E_{k_{f}}, \mathbf{k}_{\mathbf{f}}\right),
$$


where $E_{k_{i}}=k_{i}^{2} / 2$ and $E_{k_{f}}=k_{f}^{2} / 2$ are the kinetic energies of the incident electron and scattered electron, respectively, and $\mathbf{k}_{\mathbf{i}}$ and $\mathbf{k}_{\mathbf{f}}$ are their respective momenta. $\omega$ is the frequency of the considered laser, and $\ell$ is the number of photons exchanged between the electron-target system and the laser field where positive values of $\ell$ correspond to the photons' absorption and negative values to the stimulated emission of the photons.

In this scenario, we considered the helium atom to be in the ground state $1^{1} S$. The atom was excited to the $2^{1} P$ state by means of a simultaneous interaction of an electron projectile and a photon. The induced excitation process is referred to as SEPE. In general, modeling this process can be performed by evaluating the S-matrix element, expressed as:

$$
\mathbf{S}_{f, i}=-i \int_{-\infty}^{+\infty} d t\left\langle\Phi_{f}(t)\left|\hat{H}-i \frac{\partial}{\partial t}\right| \Psi_{i}(t)\right\rangle,
$$

where $\Psi_{i}(t)$ and $\Phi_{f}(t)$ are respectively the time-dependent initial and final wave functions of the electron-helium system embedded in the laser field. The operator $\hat{H}$ denotes the total Hamiltonian of the collisional system in the presence of the external laser field. The S-matrix element can be simplified in terms of the scattering amplitude $f_{e x}^{\ell}(\Delta)$ :

$$
\mathbf{S}_{f, i}=i(2 \pi)^{-1} \sum_{\ell=-\infty}^{\ell=+\infty} \delta\left(E_{k_{f}}-E_{k_{i}}+E_{f}-E_{i}-\ell \omega\right) f_{e x}^{\ell}(\boldsymbol{\Delta})
$$

which implies the conservation energy condition. $E_{i}$ and $E_{f}$ are respectively the eigenenergies associated with the helium ground state $\mathrm{He}\left(1^{1} S\right)$ and the excited state $\mathrm{He} e^{*}\left(2^{1} P\right)$ in the absence of the laser field.

In this present theoretical study, the electron-helium collision was treated within the second-order Born approximation (SBA) (i.e., the electron projectile interacted twice with the helium target), which may be more appropriate compared to the first-order Born approximation (FBA) to treat the excitation process at low impact energies. The exact wave functions describing a free electron oscillating in a laser field are known as Volkov states [35]. The wave functions describing the laser-helium interaction were calculated using the first-order time-dependent perturbation theory, which was justified as the laser intensities we considered were in the range of $0.6-2 \times 10^{7} \mathrm{~W} / \mathrm{cm}^{2}$, which were too weak to induce nonperturbative effects.

The amplitude in Equation (3), which was evaluated within the second-order Born approximation, can be expressed as:

$$
f_{e x}^{\ell}(\Delta)=f_{e x}^{B_{1}, \ell}(\Delta)+f_{e x}^{B_{2}, \ell, 0}(\boldsymbol{\Delta})
$$

where $f_{e x}^{B_{2}, \ell, 0}(\boldsymbol{\Delta})$ is the second-order Born excitation amplitude of the zeroth-order in $\mathcal{E}_{0}$ evaluated at the shifted momenta $\Delta_{\mathbf{i}}$ and $\Delta_{\mathbf{f}}$ with $\Delta_{\mathbf{i}}=\mathbf{k}_{\mathbf{i}}-\mathbf{q}$ and $\Delta_{\mathrm{f}}=\mathbf{q}-\mathbf{k}_{\mathbf{f}}$. The momentum transfer is $\Delta=\mathbf{k}_{\mathbf{f}}-\mathbf{k}_{\mathbf{i}}$, and $\mathbf{q}$ is the virtual projectile wave vector (cf. [26] and the references therein).

The excitation amplitude in Equation (4) can be expressed as:

$$
f_{e x}^{B_{2}, \ell, 0}(\boldsymbol{\Delta})=-\frac{1}{\pi^{2}} J_{\ell}(\boldsymbol{\alpha} . \boldsymbol{\Delta}) \int_{0}^{+\infty} q^{2} d q d \xi_{q}^{\prime} \frac{\left\langle\psi_{f}\left|\widetilde{V}_{d}\left(\boldsymbol{\Delta}_{f}, \mathbf{r}\right) G_{c}\left(\tilde{\zeta}^{\prime}\right) \widetilde{V}_{d}\left(\boldsymbol{\Delta}_{i}, \mathbf{r}\right)\right| \psi_{i}\right\rangle}{\Delta_{i}^{2} \Delta_{f}^{2}} .
$$

$G_{c}\left(\xi^{\prime}\right)=\sum_{n} \frac{\left|\psi_{n}\right\rangle\left\langle\psi_{n}\right|}{\xi^{\prime}-E_{n}}$ is the Coulomb-Green function with the argument $\xi^{\prime}=E_{k_{i}}+$ $E_{i}-E_{q}+\ell \omega$, and $J_{\ell}$ is an ordinary Bessel function of order $\ell$. The vector $\boldsymbol{\alpha}=\hat{\boldsymbol{\epsilon}} \mathcal{E}_{0} / \omega^{2}$ corresponds to the excursion radius of the quiver motion of the free electron in the laser field, and $\hat{\epsilon}$ is a unit vector defining the polarization direction of the electric component of the laser field. $\tilde{V}_{d}$ is the Fourier transform of the direct potential $V_{d}=-2 / r_{0}+\sum_{j=1}^{2} 1 / r_{0 j}$ where $r_{0}$ denotes the distance between the incident electron and the helium nucleus, while 
$r_{01}$ and $r_{02}$ represent the distances between the incident electron and the helium-bound electrons [26]. The wave functions $\psi_{i}, \psi_{f}$, and $\psi_{n}$ are the stationary states associated with the eigenenergies $E_{i}, E_{f}$, and $E_{n}$ of the helium target, respectively. These states were evaluated using the Sturmian approach (see [26,34,36]).

The term $f_{e x}^{B_{1}, \ell}(\Delta)$ in Equation (4) is the FBA amplitude of the excitation process associated with the exchange of $\ell$ photons. This can be given as the sum of the electronic and atomic amplitudes:

$$
f_{\text {ex }}^{B_{1}, \ell}(\boldsymbol{\Delta})=f_{\text {elec }}^{B_{1}, \ell}(\boldsymbol{\Delta})+f_{\text {atom }}^{B_{1}, \ell}(\boldsymbol{\Delta}),
$$

where the electronic term $f_{\text {elec }}^{B_{1}, \ell}(\Delta)$ describes the scattering of a Volkov electron by the bare atom, while the atomic amplitude term $f_{\text {atom }}^{B_{1}, \ell}(\boldsymbol{\Delta})$ occurs as a result of the dressing effects of the atomic target by the laser field [32].

At the low energy of the incident electron, the exchange effect is necessary for precise calculations of the differential cross-sections. This effect takes into account the fact that the electron projectile and the bound electron are indistinguishable. In this present work, we included this quantum mechanical effect in our simulations by evaluating the exchange scattering amplitude $g_{f, i}^{\ell}$ where the subscripts $i$ and $f$ denote the initial and final states, respectively (we used the same term of $g_{f, i}^{\ell}$ as in [37]). Generally, the exchange effect is more pronounced at low impact energies where the SBA is more relevant in contrast to the FBA, which is a high-impact-energy approximation [37-39]. Including the exchange, the second-Born differential cross-section (SB-DCS) corresponding to the inelastic scattering process with the transfer of $\ell$ photons reads:

$$
\frac{d \sigma_{f, i}^{\ell}}{d \Omega}=\frac{k_{f}}{k_{i}}\left|f_{f, i}^{\ell}-g_{f, i}^{\ell}\right|^{2} .
$$

which does not depend on the initial phase $\varphi$ of the laser field due to the inability of the collision time to be defined and as a result of the approximation of the projectile wave packet by a monoenergetic beam with an infinite duration [40].

Here, the generalization of the inelastic scattering of the Kroll-Watson formulation for free-free scattering in a laser field was used, where in the generalized KWA, the DCS $\frac{\mathrm{d} \sigma}{\mathrm{d} \Omega}$ on for laser-assisted scattering summed over $\ell$ is related to the field-free DCS $\frac{\mathrm{d} \sigma}{\mathrm{d} \Omega}{ }^{\text {off }}$ by [41]:

$$
\frac{\mathrm{d} \sigma^{o n}}{\mathrm{~d} \Omega}=\frac{\Delta_{i}}{\Delta_{f}} \frac{k_{f}}{k_{i}} J_{\ell}^{2}(\boldsymbol{\alpha} \cdot \boldsymbol{\Delta}){\frac{\mathrm{d} \sigma^{o f f}}{\mathrm{~d} \Omega}}^{o f}\left(\boldsymbol{\Delta}_{\mathbf{f}}, \boldsymbol{\Delta}_{\mathbf{i}}\right) .
$$

The field-free differential cross-sections were evaluated at the shifted momenta $\Delta_{\mathbf{f}}=\mathbf{k}_{\mathbf{f}}-\gamma$ and $\Delta_{\mathbf{i}}=\mathbf{k}_{\mathbf{i}}-\gamma$, where $\gamma=\ell \omega \alpha /(\alpha . \Delta)$.

\section{Results and Discussion}

The results presented in this work correspond to the reaction given by Equation (1) and were obtained for the case where the energy of the incident electron was $E_{i}=45 \mathrm{eV}$. In this scenario, the transferred energy to the atom was $E_{\text {ext }}=21.218 \mathrm{eV}=E_{i}-E_{f}$. The final state of the helium atom considered in the present work after the scattering process was the excited $H e^{*}\left(2^{1} P\right)$ state. The choice of this state was dictated by the stability of the signal. On the contrary, the measurement around the $2^{1} S$ excitation showed a greater statistical uncertainty because of the much lower field-free cross-section. (cf. Figure 1 in [13]).

Previously, Wallbank et al. [13] demonstrated the possibility of observing the electron excitation to the $2^{1} \mathrm{~S}$ and $2^{1} \mathrm{P}$ states at a small scattering angle $\left(12^{\circ}\right)$ and at an electron energy well above the threshold (i.e., $45 \mathrm{eV}$ ) with the accompanying emission and absorption of one photon of the $\mathrm{CO}_{2}$ laser. Furthermore, they predicted that the differential scattering cross-sections could be measured for SEPE for the two above-mentioned states of helium over a range of scattering angles $\left(12^{\circ}\right.$ to $\left.35^{\circ}\right)$, which has yet to be confirmed. 
In this paper, we considered a scenario in which the polarization vector of the laser field $\hat{\epsilon}$ was chosen to be parallel to the direction of the incoming electron momentum $\mathbf{k}_{i}$. The first low scattering angles for which the differential cross-section was measured were $6^{\circ}$ and $9^{\circ}$. To reach reasonable statistics yielding small error bars, the collecting data took more time, contrary to the rest of the angles. Except for their kinetic energies, the incident and the scattered electrons belonged almost to a merged beam. It is worth noting that the data obtained for all angles required several months of experimental work. We ensured that the experimental conditions for the laser, electron, and atomic beams were consistent.

In Figures 1-3, we present a comparison between the measured signals during the first three microseconds of the laser pulse and the computed signals using both the second-order Born and KWA approximations. We stress here that the duration of the laser pulse was $4 \mu \mathrm{s}$, but only the first $3 \mu$ s were considered in the detection system. The fourth microsecond was not considered since the laser intensity was weaker and unstable (cf. Figure 8 in [31]).
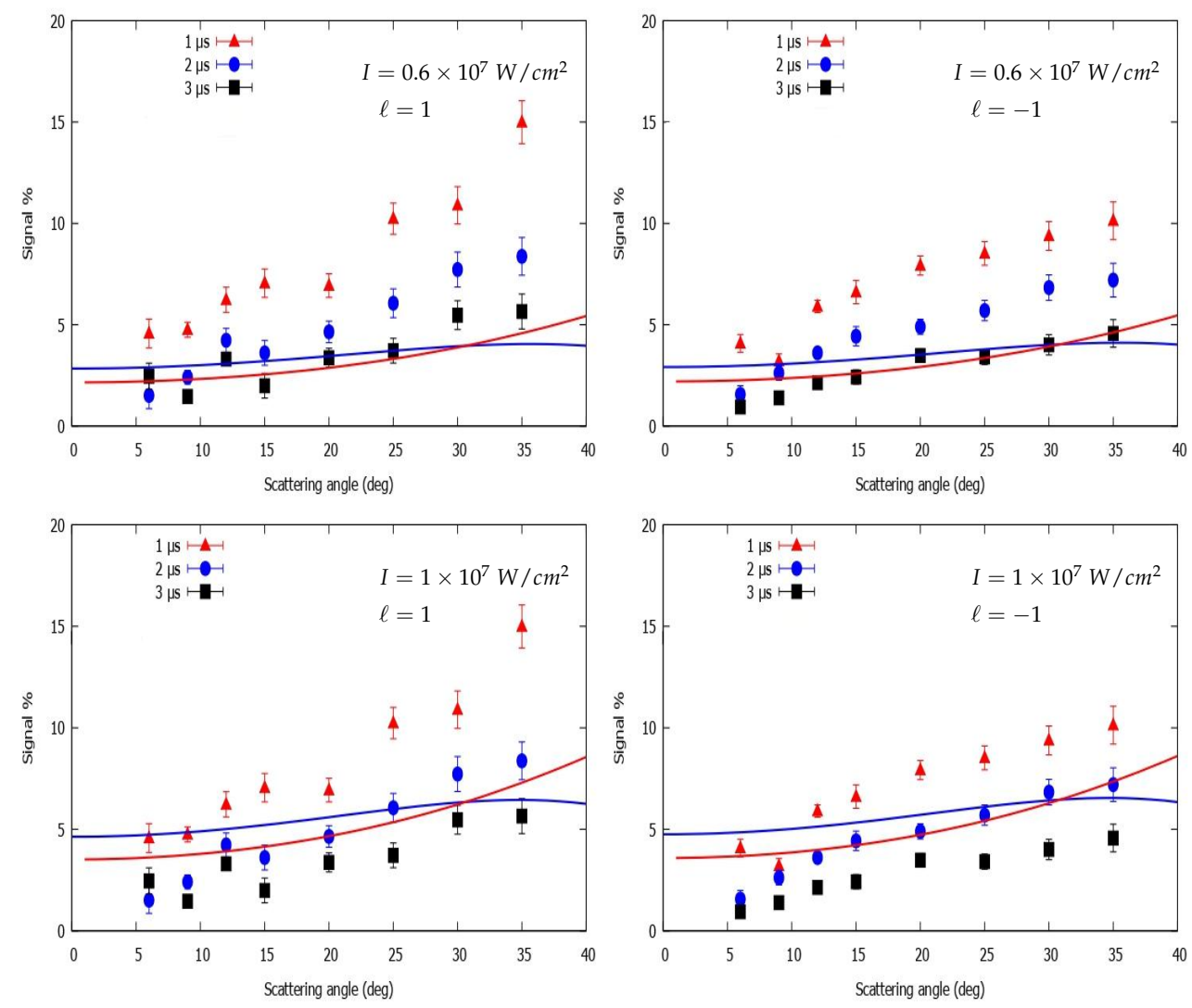

Figure 1. Signal (\%) as a function of the scattering angle. The laser intensities are $0.6 \times 10^{7} \mathrm{~W} / \mathrm{cm}^{2}$ (upper panels) and $1 \times 10^{7} \mathrm{~W} / \mathrm{cm}^{2}$ (lower panels). The left figure corresponds to the absorption of one photon $(\ell=1)$, while the right figure corresponds to the emission of one photon $(\ell=-1)$. The points correspond to the experimental results. The solid red lines correspond to the Kroll-Watson approximation results. The solid blue lines correspond to the second-order Born approximation results.

The figures are presented for the case of the absorption and emission of one photon of $117 \mathrm{meV}$ and are displayed at different peak intensities: $0.6 \times 10^{7}, 1 \times 10^{7}, 1.5 \times 10^{7}$, and $2 \times 10^{7} \mathrm{~W} / \mathrm{cm}^{2}$. The measured and computed signals correspond to the ratios of the SEPE signal when the laser was on the field-free signal, expressed as a percentage of the latter. Here, we did not consider the scenario involving the absorption and emission of two photons, as the intensity of the signals was too weak. 

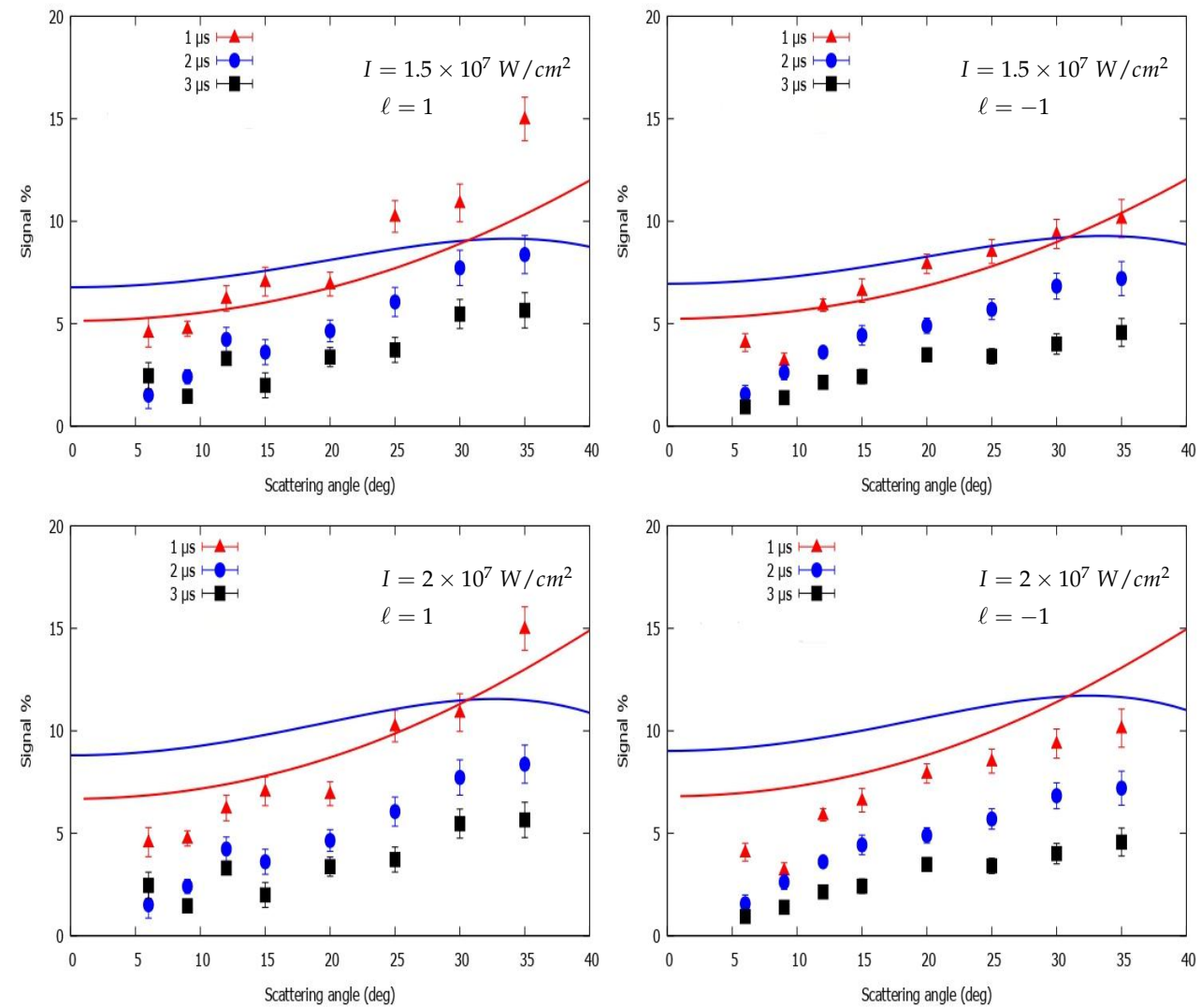

Figure 2. Same as Figure 1, but for laser intensities of $1.5 \times 10^{7} \mathrm{~W} / \mathrm{cm}^{2}$ (upper panels) and $2 \times 10^{7} \mathrm{~W} / \mathrm{cm}^{2}($ lower panels).
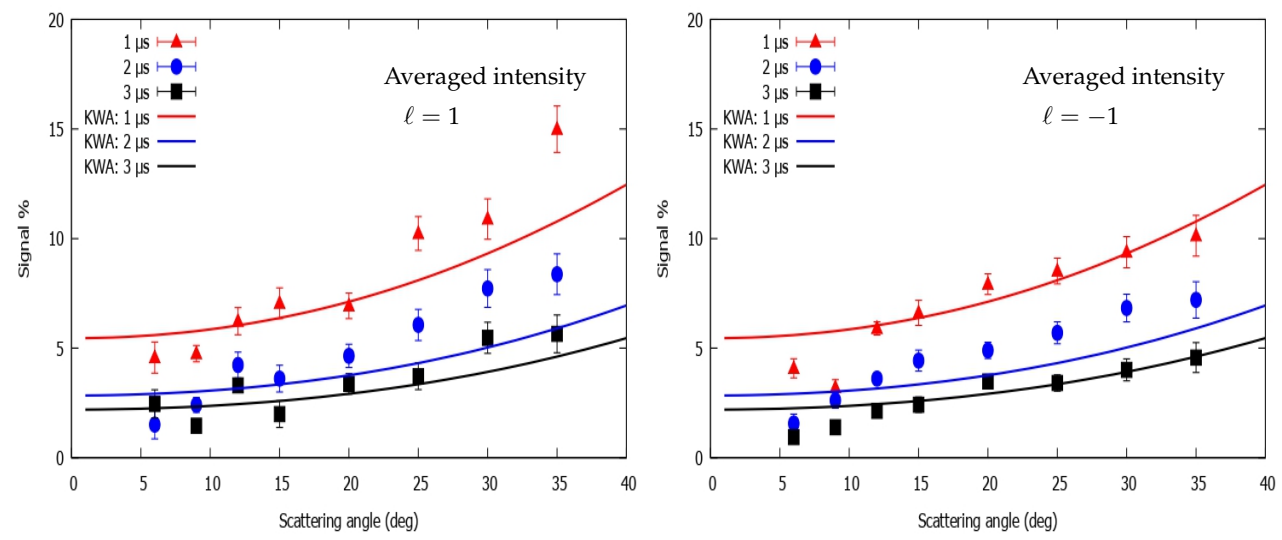

Figure 3. Signal (\%) as a function of the scattering angle. The left figure corresponds to the absorption of one photon $(\ell=1)$, while the right figure corresponds to the emission of one photon $(\ell=-1)$. The points correspond to the experimental results, and the solid lines correspond to the Kroll-Watson approximation results when the laser intensity was averaged.

The measured signals exhibited the same behavior for the absorption and emission of the photon and for the $3 \mu$ s of the laser, which were manifested as an increase of the signal as a function of the scattering angle. A closer inspection of these measured signals indicated some distinct features that were found to be sensitive to the nature of the exchanged photons. This sensitivity however was seen to be less significant in the computed signals using both the SBA and KWA methods. Furthermore, in the experiment, the signals showed the emergence of a structure at a small scattering angle of around $15^{\circ}$, which was absent in the theoretical considerations. This region encoded important information about the scattering process, in particular the laser-induced dressed states [24,25]. Here, an accurate numerical description of the three interactions involved in the scattering process in this 
region is necessary in order to produce the experimental findings and to interpret them properly. Most of the comparisons were based on the application of the Kroll-Watson approximation [42], in which a disagreement with the experiment at small scattering angles for elastic and inelastic scattering was found [15-17].

In general, the scattering amplitude describing the electron-atom collisions in the presence of the laser field was considered to have two contributions: an electronic contribution that corresponded to the interaction of the electron with the laser field and an atomic contribution that occurred through the atomic wave functions being dressed by the laser field. At small scattering angles, the two amplitudes might be of the same order of magnitude, which would leads to interference effects that would manifest in the differential cross-sections as an oscillatory structure. This structure was found to be sensitive to the duration of the laser pulse. This sensitivity suggested the build-up of the interference effects in the time domain.

In the Kroll-Watson treatment, the atomic contribution was neglected, but it has been shown that, at small scattering angles, this contribution is significant and may lead to interferences, either constructive or destructive, between the two contributions of the scattering amplitude [13]. An accurate description of these contributions is therefore a necessity in order to produce these interference effects. Here, although these contributions were taken into account in our treatment based on the SBA, our approach failed to reproduce the structure that emerged at small scattering angles in the experiment.

On the other hand, in order to reproduce the measured signals accurately, the conditions of the overlap between the three beams should be included in the calculations. It has been shown that improving the accuracy of the calculations requires taking into consideration the spatial and temporal distributions of the three beams involved in the scattering process $[43,44]$. Here, we performed a cubic interpolation of the temporal laser profile in the KWA calculation to average the signal. The best agreement of the shape of the signal was found when the laser intensity was averaged, as shown in Figure 3. In a former paper [31], it was shown that the misalignment in the region of the interaction among the three beams was responsible for the shift between the theory and experiments. We note that the temporal averaging of the laser profile was not considered in the SBA calculations. This was mainly because of the complexity of the S-matrix when it was treated in the second-order approximation and when the three interactions (i.e., photon, electron, and target), as well as the atomic structure were taken into account. This however was not the case in the KWA. On the other hand, the simplicity of the expression of the KWA allowed us to show this temporal dependence, which we believe provided the necessary insights to complement the experimental findings.

At this point, we concluded that although our theoretical calculations reproduced the general shape of the measured signals very well, they failed to reproduce them precisely at small scattering angles. This calls for new accurate calculations beyond the Born series to further understand the origin of the structure that emerged. We thus considered our work as a benchmark study to stimulate other theoretical works to address this issue with more sophisticated theoretical approaches.

\section{Conclusions}

We presented a comparative study of an experiment and a theory of the simultaneous electron-photon excitation (SEPE) of the $\mathrm{He}^{1} \mathrm{P}$ state. We measured the signals during the first three microseconds of the laser pulse for a scenario involving the absorption and emission of one photon and for a monoenergetic electron beam of $45 \mathrm{eV}$. Our theoretical approaches were based on the second-order Born and Kroll-Watson approximations. We found a good agreement with the experiment except at a small scattering angle, for which our calculations failed to reproduce the structure that emerged in the experiment. In this region, different contributions of the laser-assisted scattering process may emerge with comparative magnitudes, which might result in constructive or destructive interferences. Our suggestion calls for additional accurate calculations to further understand the origin 
of this structure. Our study, therefore, reveals a hidden aspect in laser-assisted scattering, which will definitively stimulate further theoretical works to address the SEPE process in other systems.

Author Contributions: I.A. performed the numerical calculations. D.N. carried out the experiment and wrote the first draft. D.K. and A.M. developed the software. A.T. and A.M. reviewed and edited the manuscript. H.A. re-wrote the entire manuscript. All authors have read and agreed to the published version of the manuscript.

Funding: This research received no external funding.

Institutional Review Board Statement: Not applicable.

Informed Consent Statement: Not applicable.

Data Availability Statement: The data that support the findings of this study are available from the corresponding author upon reasonable request.

Acknowledgments: We would like to thank J.K. Holmes (from St. Francis Xavier University, Dept. of Physics, Antigonish) for useful help in achieving the measurements and the Natural Sciences and Engineering Research Council of Canada and the St Francis Xavier University Council for Research for financial support.

Conflicts of Interest: The authors declare no conflict of interest.

\section{References}

1. Corkum, P.; Krausz, F. Attosecond science. Nat. Phys. 2007, 3, 381. [CrossRef]

2. Krausz, F.; Ivanov, M. Attosecond physics. Rev. Mod. Phys. 2009, 81, 163. [CrossRef]

3. Corkum, P.B. Plasma perspective on strong field multiphoton ionization. Phys. Rev. Lett. 1993, 71, 1994. [CrossRef]

4. Hentschel, M.; Kienberger, R.; Spielmann, C.; Reider, G.A.; Milosevic, N.; Brabec, T.; Corkum, P.; Heinzmann, U.; Drescher, M.; Krausz, F. Attosecond metrology. Nature 2001, 414, 509. [CrossRef]

5. Rudenko, A.; de Jesus, V.L.B.; Ergler, T.; Zrost, K.; Feuerstein, B.; Schröter, C.D.; Moshammer, R.; Ullrich, J. Correlated TwoElectron Momentum Spectra for Strong-Field Nonsequential Double Ionization of He at $800 \mathrm{~nm}$. Phys. Rev. Lett. 2007, 99 , 263003. [CrossRef] [PubMed]

6. Chen, Z.; Liang, Y.; Lin, C.D. Quantum Theory of Recollisional (e,2e) Process in Strong Field Nonsequential Double Ionization of Helium. Phys. Rev. Lett. 2010, 104, 253201. [CrossRef] [PubMed]

7. Mason, N.J.; Newell, W.R. Simultaneous electron-photon excitation of the helium $2^{3}$ S state. J. Phys. B At. Mol. Phys. 1987, 20, L323. [CrossRef]

8. Mason, N.J.; Newell, W.R. Simultaneous electron-photon excitation of the helium $2^{3}$ S state. J. Phys. B At. Mol. Opt. Phys. 1989, 22, 777. [CrossRef]

9. Wallbank, B.; Holmes, J.K.; LeBlanc, L.; Weingartshofer, A. Simultaneous off-shell excitation of He $2^{3} \mathrm{~S}$ by an electron and one or more photons. Z. Phys. D 1988, 10, 467. [CrossRef]

10. Wallbank, B.; Holmes, J.K.; Weingartshofer, A. Simultaneous electron-photon excitation of He $2^{3}$ S: An experimental investigation of the effects of laser intensity and polarisation. J. Phys. B At. Mol. Opt. Phys. 1990, 23, 2997. [CrossRef]

11. Luan, S.; Hippler, R.; Lutz, H.O. Simultaneous electron-photon excitation of helium ( $\hbar \omega=1.17$ eV). J. Phys. B At. Mol. Opt. Phys. 1991, 24, 3241. [CrossRef]

12. Wallbank, B.; Holmes, J.K.; Weingartshofer, A. Experimental differential cross sections for multiphoton free-free transitions. J. Phys. B At. Mol. Phys. 1987, 20, 6121-6138. [CrossRef]

13. Wallbank, B.; Holmes, J.K.; Weingartshofer, A. Absorption and emission of radiation during electron excitation of the $2^{1} \mathrm{~S}$ and $2^{1} \mathrm{P}$ states of helium. Phys. Rev. A 1989, 40, 5461-5463. [CrossRef]

14. Wallbank, B.; Holmes, J.K.; Weingartshofer, A. Simultaneous electron-photon excitation of metastable states of rare-gas atoms. J. Phys. B At. Mol. Opt. Phys. 1989, 22, L615. [CrossRef]

15. Wallbank, B.; Holmes, J.K. Laser-assisted elastic electron-atom collisions. Phys. Rev. A 1993, 48, R2515-R2518. [CrossRef]

16. Wallbank, B.; Holmes, J.K. Laser-assisted elastic electron-atom collisions: Low electron energy and small scattering angle. J. Phys. B At. Mol. Opt. Phys. 1994, 27, 1221-1231. [CrossRef]

17. Wallbank, B.; Holmes, J.K. Differential cross sections for laser-assisted elastic electron scattering from argon. J. Phys. B At. Mol. Opt. Phys. 1994, 27, 5405-5418. [CrossRef]

18. Wallbank, B.; Holmes, J.K. Low-energy electron-helium scattering in a laser field. J. Phys. B At. Mol. Opt. Phys. 1996, 29, 5881. [CrossRef]

19. Wallbank, B.; Holmes, J.K. Laser-assisted elastic electron scattering from helium. Can. J. Phys. 2001, 79, $1237-1246$.

20. Makhoute, A.; Agueny, A.; Dubois, A.; Ajana, I.; Taoutioui, A. Electron-impact elastic scattering of helium in the presence of a laser field: Non perturbative approach. J. Phys. B At. Mol. Phys. 2016, 49, 075204. [CrossRef] 
21. Zaytsev, A.S.; Zaytsev, S.A.; Ancarani, L.U.; Kouzakov, K.A. Laser-modified Coulomb scattering states of an electron in the parabolic quasi-Sturmian-Floquet approach. Phys. Rev. A 2018, 97, 043417. [CrossRef]

22. Makhoute, A.; Khalil, D.; Ajana, I. Laser-assisted (e,2e) collisions in the symmetric/asymmetric coplanar geometry. Atoms 2019, 7, 40. [CrossRef]

23. Agueny, H.; Makhoute, A.; Dubois, A.; Ajana, I.; Rahali, G. Laser-assisted inelastic scattering of electrons by helium atoms. Phys. Rev. A 2015, 92, 013423. [CrossRef]

24. Agueny, H.; Makhoute, A.; Dubois, A. Dynamic-Stark-effect-induced coherent mixture of virtual paths in laser-dressed helium: Energetic electron impact excitation. J. Phys. B At. Mol. Phys. 2017, 50, 125002. [CrossRef]

25. Agueny, H.; Makhoute, A. Monoenergetic electron-beam stimulated light-dressing-induced resonance in a helium atom. J. Phys. B At. Mol. Phys. 2018, 51, 025003. [CrossRef]

26. Ajana, I.; Makhoute, A.; Khalil, D. Low-energy electron-helium scattering in a Nd-YAG laser field. J. Electron. Spectrosc. Relat. Phenom. 2014, 192, 19. [CrossRef]

27. Ajana, I.; Makhoute, A.; Khalil, D.; Chaddou, S. Exchange effects and second-order Born corrections in laser-assisted (e,2e) collisions with helium atoms. Phys. Rev. A 2015, 91, 043411. [CrossRef]

28. Dunseath, K.M.; Terao-Dunseath, M. Simultaneous electron-photon excitation of helium in a $\mathrm{CO}_{2}$ laser field. J. Phys. B At. Mol. Phys. 2011, 44, 135203. [CrossRef]

29. Dunseath, K.M.; Terao-Dunseath, M. Simultaneous electron-photon excitation of helium in an Nd: YAG laser field. J. Phys. B At. Mol. Phys. 2013, 46, 235201. [CrossRef]

30. Francken, P.; Joachain, C.J. Electron-impact excitation of atoms in the presence of a nearly resonant laser field. Phys. Rev. A 1990, 41, 3770. [CrossRef] [PubMed]

31. Nehari, D.; Holmes, A.J.K.; Dunseath, K.M.; Terao-Dunseath, M. Experimental and theoretical study of free-free electron-helium scattering in a $\mathrm{CO}_{2}$ laser field. J. Phys. B At. Mol. Phys. 2010, 43, 025203. [CrossRef]

32. El Akramine, O.; Makhoute, A.; Khalil, D.; Maquet, A.; Taïeb, R. Effects of laser polarization in laser-assisted electron-helium inelastic collisions: A Sturmian approach. J. Phys. B At Mol. Opt. Phys. 1999, 32, 2783 [CrossRef]

33. Bouzidi, M.; Makhoute, A.; Khalil, D.; Maquet, A.; Joachain, C.J. On the second Born approximation for laser-assisted electronatom collisions. J. Phys. B At. Mol. Opt. Phys. 2001, 34, 737. [CrossRef]

34. Makhoute, A.; Khalil, D.; Zitane, M.; Bouzidi, M. The second Born approximation in electron-atom collisions in the presence of a laser field. J. Phys. B At. Mol. Opt. Phys. 2002, 35, 957. [CrossRef]

35. Volkov, D.M. On a class of solutions of the Dirac equation. Z. Phys. 1935, 94, 50.

36. Bouzidi, M.; Makhoute, A.; Hounkounou, M.N. Polarisation effect of laser field in inelastic electron - hydrogen collisions. Eur. Phys. J. D 1999, 5, 159. [CrossRef]

37. Byron, F.W.; Joachain, C.J. Correlation effects in atoms. I. Helium. Phys. Rev. 1966, 146, 1. [CrossRef]

38. Francken, P.; Attaourti, Y.; Joachain, C.J. Laser-assisted inelastic electron-atom collisions. Phys. Rev. A 1988, 38, 1785. [CrossRef]

39. Sun, J.; Zhang, S.; Jiang, Y.; Yu, G. Electron-atom scattering at small angles in a $\mathrm{CO}_{2}$ laser field Phys. Rev. A 1998, 94, 2225. [CrossRef]

40. Dörr, M.; Joachain, C.J.; Potvliege, R.M.; Vuc̃ic, S. Born-Floquet theory of laser-assisted electron-atom collisions. Phys. Rev. A 1994, 49, 4852. [CrossRef]

41. Mittleman, M.H. Electron-atom scattering in the field of a low-frequency laser. Phys. Rev. A 1980, 21, 79. [CrossRef]

42. Kroll, N.M.; Watson, K.M. Charged-particle scattering in the presence of a strong electromagnetic wave. Phys. Rev. A 1973, 8, 804. [CrossRef]

43. Bivona, S.; Burlon, R.; Zangaran, R.; Ferrante, G. Electron scattering in strong laser fields. Theoretical models versus recent experiments. J. Phys. B At. Mol. Phys. 1985, 18, 3149. [CrossRef]

44. Weingartshofer, A.; Holmes, J.K.; Sabbagh, J.; Chin, S.L. Electron scattering in intense laser fields. J. Phys. B At. Mol. Phys. 1983, 16, 1805. [CrossRef] 